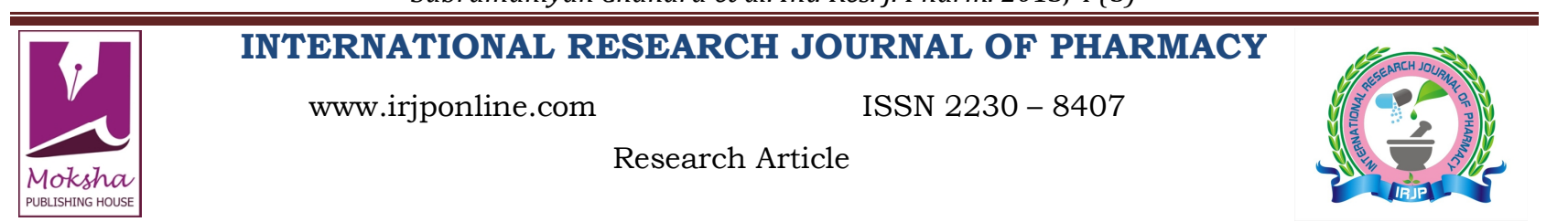

\title{
DESIGN AND IN VITRO EVALUATION STUDIES OF DICLOFENAC SODIUM FLOATING MATRIX TABLETS BY MELT GRANULATION TECHNIQUE
}

Subramaniyan Chandra*, S.Gopi, Biyogo ogetii alex, Omarbakri nasreidin elsayed, Oleti Chandra sai pavan Department of Pharmaceutics, J.K.K. Munirajah College of Pharmacy, Komarapalayam, Nammakal, India

Email: chandrajkkm@gmail.com

Article Received on: 19/02/13 Revised on: 01/03/13 Approved for publication: 17/04/13

DOI: $\mathbf{1 0 . 7 8 9 7 / 2 2 3 0 - 8 4 0 7 . 0 4 5 3 1}$

IRJP is an official publication of Moksha Publishing House. Website: www.mokshaph.com

(C) All rights reserved.

\begin{abstract}
The purpose of this study was to formulate diclofenac sodium floating matrix drug delivery system by melt granulation technique. These tablets were developed to prolong gastric residence time and increase its bio availability. The diclofenac sodium tablets of $100 \mathrm{mg}$ were developed by using different bees wax combinations which are prepared by melt granulation technique by using polymers such as Hydroxy propyl methyl cellulose(HPMC K15M), ethyl cellulose, bees wax alone or in combination with cetyl alcohol, sodium bicarbonate as a gas generating agent and other standard excipients. The prepared granules are subjected to pre compression studies such as angle of repose, bulk density, tapped density, compressibility index, Hausner's ratio. The optimized formulation produced monolithic tablets with optimum hardness, uniform thickness, consistent weight uniformity and low friability. The results of dissolution studies, floating lag time indicates that formulations F6 exhibited good and controlled drug release .

Key words: Diclofenac sodium, Melt granulation, Dissolution, $0.1 \mathrm{~N} \mathrm{HCl}$
\end{abstract}

\section{INTRODUCTION}

Floating drug delivery systems were first described by Davis in 1968. These systems were used to prolong the gastric residence time of drug delivery systems. They remain buoyant in the stomach for prolonged period of time without affecting the gastric emptying rate of other contents. A floating dosage form is useful for those drugs that act locally in the proximal gastro intestinal tract are unstable in lower parts of gut or are poorly absorbed in the intestine ${ }^{1-3}$.

Diclofenac sodium is a nonsteroidal anti infalammatory drugs having chemical name sodium 2-[(2,6-dichlorophenyl)amino] Phenyl acetate. The most frequent adverse side effects occurring with diclofenac sodium are gastro intestinal disturbances, peptic ulceration and gastro intestinal bleeding, hence there is potential need for a floating matrix dosage form for this drug to minimize gastric erosion side effect. It's biological half life on the other hand is very short, sustaining its anti inflammatory activity only for a few hours and associated adverse effects; it is considered as an ideal model drug for floating matrix drug delivery ${ }^{4-10}$. The present study is to formulate rate controlled floating drug delivery system of diclofenac sodium which is primarily absorbed from stomach, so by increasing the GRT of the drug, the bio availability can be increased. As the moderately weekly acidic drug, so the stomach is the major absorption site for the drug. So basing on this fact the drug is made to float in the stomach using mechanism of buoyancy by using gas generating agent and the drug is delivered in the sustained release dosage form by using HPMC as swell able polymer to formation of gel layer for controlled release. Ethyl cellulose is used to retard the disintegration and also dissolution. Microcrystalline cellulose is used as diluents ${ }^{11}$. The study also includes various evaluation studies of diclofenac sodium tablet and the effect of processing variables on it. A possible interaction between drug and polmers are also investigated by FTIR study.

The objective of this study is to formulate diclofenac sodium floating matrix tablets using hydrophobic wax materials, sodium bicarbonate as gas generating agent and to evaluate the in vitro release characteristics and to predict and correlate the release behavior of diclofenac from the matrix

\section{MATERIALS AND METHODS \\ Materials}

Diclofenac sodium as a gift of hetero chemicals Ltd, Hyderabad, HPMC K15M by SD fine chemicals, Mumbai, Ethyl cellulose from Loba chemicals Pvt Ltd, Mumbai, Micro crystalline cellulose and talc from Yarrow chemicals Ltd, Mumbai. Sodium bicarbonate, Magnesium stearate for Thomas bakers Ltd, Mumbai

\section{Methods}

Preparation of standard curve of diclofenac sodium

Solutions ranging fron 2 to 10 microgram per $\mathrm{ml}$ were prepared by using ethanol and $0.1 \mathrm{~N} \mathrm{HCl}$ absorbance was measured for each solution at lambda max of 276nm, using Shimadzu spectrophotometer. Correlation coefficient was found to be 0.9996 in $0.1 \mathrm{~N} \mathrm{HCl}$.

\section{Preparation of floating tablets by melt granulation technique}

Floating tablets, each containing $100 \mathrm{mg}$ diclofenac were formulated by a conventional melt technique. The composition of various formulations of the tablets with their codes is listed in table. The composition with respect to polymer combination was selected on the basis of trial preparation of tablets. The amount of bees wax was decreased gradually and the reduced amount of bees wax was replaced by cetyl alcohol. As per each formulation batch code required quantity of bees wax and cetyl alcohol were weighed and melted separately in a large china dish over a water bath. The drug was added to molten wax and mixed well. Previously weighed quantities of ethyl cellulose, HPMC, Micro crystalline cellulose and sodium carbonate were added to the drug wax mixture and mixed well. After through mixing china dish was removed from water bath and cooled. The coherent mass was then scrapped from the china dish and was passed through sieve no 30 . The amount of ingredients that are added for each tablet is given in following table 1 . 
Evaluation of granules

Angle of repose

Flowability of different batch of granules was determined by calculating angle of repose by fixed height method. A funnel with $10 \mathrm{~mm}$ inner diameter of stem was fixed at a height of 2 $\mathrm{cm}$ over the platform. About $10 \mathrm{gm}$ of sample was slowly passed along the wall of the funnel till the tip of the pile formed and touches the stem of the funnel. A rough circle was drawn around the pile base and the radius of the powder cone was measured.

Angle of repose was calculated from the average radius using the following formula.

$$
\Theta=\tan ^{-1}(\mathbf{h} / \mathbf{r})
$$

Where, $\Theta=$ Angle of repose, $\mathrm{h}=$ height of the pile, $\mathrm{r}==$ average radius of the powder cone

\section{Bulk density}

Apparent bulk density was determined by pouring preserved drug excipient blend in to graduated cylinder and measuring the volume and weight "as it is". It is expressed in $\mathrm{g} / \mathrm{ml}$ and is given by

$$
\mathbf{D}_{\mathbf{b}}=\mathbf{M} / \mathbf{V o}
$$

Where, $\mathrm{M}$ is the mass of powder and $\mathrm{V}_{\mathrm{o}}$ is the bulk volume of the powder

\section{Tapped density}

It was determined by placing a graduated cylinder, containing a known mass of drug excipients blend, on mechanical tapping apparatus. the tapped volume was measured by tapping the powder to constant volume. It is expressed in $\mathrm{g} / \mathrm{ml}$ and is given by

$$
\mathbf{D}_{\mathrm{t}}=\mathbf{M} / \mathbf{V}_{\mathrm{t}}
$$

Where $\mathrm{M}$ is the mass of powder and $\mathrm{V}_{\mathrm{t}}$ is the tapped volume of the powder

\section{Powder flow properties}

The flow properties were determined by

\section{Carr's index(i)}

It is expressed in percentage and is expressed by

$$
\mathbf{I}=\mathbf{D}_{\mathbf{t}}-\mathbf{D}_{\mathrm{b}} / \mathbf{D}_{\mathbf{t}}
$$

Where $D_{t}$ is the tapped density of the powder and $D_{b}$ bulk density of the powder

\section{Hausner's ratio}

It is expressed in percentage and is expressed by

$$
\mathbf{H}=\mathbf{D}_{\mathbf{t}} / \mathbf{D}_{\mathrm{b}}
$$

Where $D_{t}$ is the tapped density of the powder and $D_{b}$ is the bulk density of the powder

\section{Compression of tablets}

After evaluation of granules were then compressed in to the tablet by direct compression technique using 10 punches tablet punching machine

\section{Evaluation of tablets}

Weight variation

20 tablets were selected at random and average weights were determined. The individual tablets weighed the individual weight was compared with the average.

\section{Thickness and diameter}

The thickness and diameter of the tablets was measured by Vernier calipers. It is expressed in $\mathrm{mm}$.

\section{Hardness}

The hardness of the tablet was determined using a Monsanto hardness tester. It is expressed in $\mathrm{kg} / \mathrm{cm}^{2}$.

\section{Friability (f)}

The friability of the tablet was determined using roche friabilator. The friabilator was operated at $25 \mathrm{Rpm}$ per min for $4 \mathrm{~min}$ (100 revolutions). The tablets were weighed again $\left(\mathrm{W}_{\text {final }}\right)$. The $\%$ friability was then calculated by

$$
\mathbf{F}=\mathbf{W}_{\text {initial }}-\mathbf{W}_{\text {final }} / \mathbf{W}_{\text {initial }} * \mathbf{1 0 0}
$$

\section{Content uniformity}

20 tablets were taken and amount of drug present in each tablet was determined. The tablets were crushed in a mortar and the powder equivalent $100 \mathrm{mg}$ of drug was transferred to $100 \mathrm{ml}$ standard flask. The powder was dissolved in $5 \mathrm{ml}$ of methanol and make up to volume with $0.1 \mathrm{~N} \mathrm{HCl}$. The sample was mixed thoroughly and filtered through $0.45 \mu$ membrane filter. The filtered solution was diluted suitably and analyzed for drug content by UV spectrophotometer at lambda max of 276nm using $0.1 \mathrm{~N} \mathrm{HCL}$ as blank.

\section{In vitro buoyancy studies}

The randomly selected tablets from each formulation were kept in a $100 \mathrm{ml}$ beaker containing simulated gastric fluid $\mathrm{pH}$ 1.2 as per USP. The time taken for the tablet to rise to the surface and float was taken as a floating lag time. the duration of time the dosage form constantly remained on the surface of medium was determined as the total floating time.

\section{In vitro dissolution studies}

The dissolution test was performed using $900 \mathrm{ml}$ of $0.1 \mathrm{~N}$ $\mathrm{HCL}$ at $37 \pm 0.5^{\circ} \mathrm{c}$ and $75 \mathrm{rpm}$. A sample $(5 \mathrm{ml})$ of the solution was withdrawn from the dissolution apparatus hourly and samples were replaced with fresh dissolution medium. The samples were filtered through a 0.45 microns membrane filter and diluted to a suitable concentration with 0.1N HCL. Absorbance of these solutions were measured at 276nm using a Shimadzu UV spectrophotometer.

\section{RESULTS AND DISCUSSION}

Diclofenac sodium is a water insoluble drug. Its poor inherent compressibility coupled with associated side effect possess a significant challenge for developing floating tablets. For developing floating tablets with desirable drug release profile, cost effectiveness and broader regulatory acceptance combination of HPMC, Ethyl cellulose, Bees wax and Cetyl alcohol was chosen as release controlling polymers, sodium bicarbonate was added as a gas generating agent.

The formulated granule blends of different formulations (F1 to F10) were evaluated for angle of repose, tapped density, bulk density, Carr's index and Hausner's ratio. 
Subramaniyan Chandra et al. Int. Res. J. Pharm. 2013, 4 (5)

TABLE 1: FORMUALTION INGREDIENTS FOR FLOATING MATRIX TABLETS OF DICLOFENAC SODIUM

\begin{tabular}{|c|c|c|c|c|c|c|c|c|c|c|}
\hline Ingredients & $\mathrm{F} 1$ & $\mathrm{~F} 2$ & F3 & $\mathrm{F} 4$ & F5 & F6 & F7 & $\mathrm{F} 8$ & F9 & $\mathrm{F} 10$ \\
\hline Diclofenac sodium & 100 & 100 & 100 & 100 & 100 & 100 & 100 & 100 & 100 & 100 \\
\hline Bees wax & 90 & 90 & 90 & 90 & 90 & 70 & 60 & 50 & 40 & 35 \\
\hline Cetyl alcohol & - & - & - & - & - & 20 & 30 & 40 & 50 & 55 \\
\hline HPMC K15M & 64 & 64 & 64 & 64 & 64 & 64 & 64 & 64 & 64 & 64 \\
\hline Ethyl cellulose & 30 & 30 & 30 & 30 & 30 & 30 & 30 & 30 & 30 & 30 \\
\hline Microcrystallinecellulose & 60 & 50 & 40 & 35 & 30 & 30 & 30 & 30 & 30 & 30 \\
\hline Sodium bicarbonate & - & 10 & 20 & 25 & 30 & 30 & 30 & 30 & 30 & 30 \\
\hline Magnesium stearate & 3 & 3 & 3 & 3 & 3 & 3 & 3 & 3 & 3 & 3 \\
\hline talc & 3 & 3 & 3 & 3 & 3 & 3 & 3 & 3 & 3 & 3 \\
\hline Total weight of tablet in $\mathrm{mg}$ & 350 & 350 & 350 & 350 & 350 & 350 & 350 & 350 & 350 & 350 \\
\hline
\end{tabular}

TABLE 2: PRE COMPRESSION PARAMETERS OF FORMULATED GRANULES

\begin{tabular}{|c|c|c|c|c|c|}
\hline Formulation code & Angle of repose in degree & Bulk density & Tapped density & Carr's index & Hausner's ratio \\
\hline F1 & 15.2 & 0.57 & 0.71 & 19.0 & 1.24 \\
\hline F2 & 12.4 & 0.55 & 0.67 & 16.9 & 1.22 \\
\hline F3 & 10.2 & 0.55 & 0.70 & 19.9 & 1.27 \\
\hline F4 & 14.0 & 0.54 & 0.73 & 21.5 & 1.35 \\
\hline F5 & 17.7 & 0.53 & 0.67 & 20.8 & 1.26 \\
\hline F6 & $\mathbf{1 6 . 6}$ & $\mathbf{0 . 5 7}$ & $\mathbf{0 . 7 4}$ & $\mathbf{2 3 . 1}$ & 1.29 \\
\hline F7 & 19.2 & 0.56 & 0.74 & 23.7 & 1.30 \\
\hline F8 & 18.7 & 0.57 & 0.73 & 22.8 & 1.32 \\
\hline F9 & 20.8 & 0.58 & 0.72 & 18.7 & 1.24 \\
\hline F10 & 20.3 & 0.55 & 0.71 & 18.9 & 1.24 \\
\hline
\end{tabular}

TABLE 3: POST COMPRESSION PROPERTIES OF FORMULATED FLOATING TABLETS

\begin{tabular}{|c|c|c|c|c|c|c|c|}
\hline Formulation code & $\begin{array}{c}\% \text { of weight } \\
\text { variation }\end{array}$ & $\begin{array}{l}\text { Thickness } \\
\text { (mm) }\end{array}$ & Diameter & hardness & Friability & $\begin{array}{c}\text { Drug } \\
\text { content }\end{array}$ & $\begin{array}{l}\% \text { Drug } \\
\text { release }\end{array}$ \\
\hline F1 & $350 \pm 4$ & $5.3 \pm 0.01$ & $9.9 \pm 0.01$ & $4.0 \pm 0.01$ & $0.28 \pm 0.01$ & 96.6 & 69.02 \\
\hline $\mathrm{F} 2$ & $350 \pm 5$ & $5.5 \pm 0.01$ & $9.9 \pm 0.01$ & $4.9 \pm 0.01$ & $0.22 \pm 0.01$ & 96.1 & 70.20 \\
\hline F3 & $350 \pm 4$ & $5.3 \pm 0.01$ & $9.9 \pm 0.01$ & $4.9 \pm 0.01$ & $0.18 \pm 0.01$ & 97.0 & 71.13 \\
\hline F4 & $350 \pm 3$ & $5.3 \pm 0.01$ & $9.9 \pm 0.01$ & $4.9 \pm 0.01$ & $0.44 \pm 0.01$ & 96.1 & 72.23 \\
\hline F5 & $350 \pm 2$ & $5.4 \pm 0.01$ & $9.9 \pm 0.02$ & $4.4 \pm 0.01$ & $0.38 \pm 0.01$ & 96.1 & 72.21 \\
\hline F6 & $350 \pm 4$ & $5.2 \pm 0.01$ & $9.9 \pm 0.01$ & $5.0 \pm 0.01$ & $0.49 \pm 0.01$ & 95.6 & 75.02 \\
\hline F7 & $350 \pm 3$ & $5.5 \pm 0.01$ & $9.9 \pm 0.02$ & $5.0 \pm 0.01$ & $0.41 \pm 0.01$ & 97.0 & 59.85 \\
\hline F8 & $350 \pm 4$ & $5.3 \pm 0.01$ & $9.9 \pm 0.01$ & $5.0 \pm 0.01$ & $0.28 \pm 0.01$ & 96.6 & 58.52 \\
\hline F9 & $350 \pm 3$ & $5.4 \pm 0.01$ & $9.9 \pm 0.02$ & $5.0 \pm 0.01$ & $0.25 \pm 0.01$ & 95.6 & 58.07 \\
\hline F10 & $350 \pm 4$ & $5.3 \pm 0.01$ & $9.9 \pm 0.01$ & $5.0 \pm 0.01$ & $0.28 \pm 0.01$ & 96.6 & 57.96 \\
\hline
\end{tabular}

TABLE 4: IN VITRO BUOYANCY STUDIES RESULTS OF DICLOFENAC SODIUM FLOATING MATRIX TABLETS

\begin{tabular}{|c|c|c|c|}
\hline Formulation code & Amount of sodium bicarbonate in mg & Buoyancy lag time in min & Total floating time in hrs \\
\hline F1 & 0 & Did not float & did not float \\
\hline F2 & 10 & 30 & $>4$ \\
\hline F3 & 20 & 18 & $>6$ \\
\hline F4 & 25 & 5 & $>8$ \\
\hline F5 & 30 & $\mathbf{5}$ & $>12$ \\
\hline F6 & $\mathbf{3 0}$ & 5 & $>12$ \\
\hline F7 & 30 & 5 & $>12$ \\
\hline F8 & 30 & 4 & \\
\hline
\end{tabular}




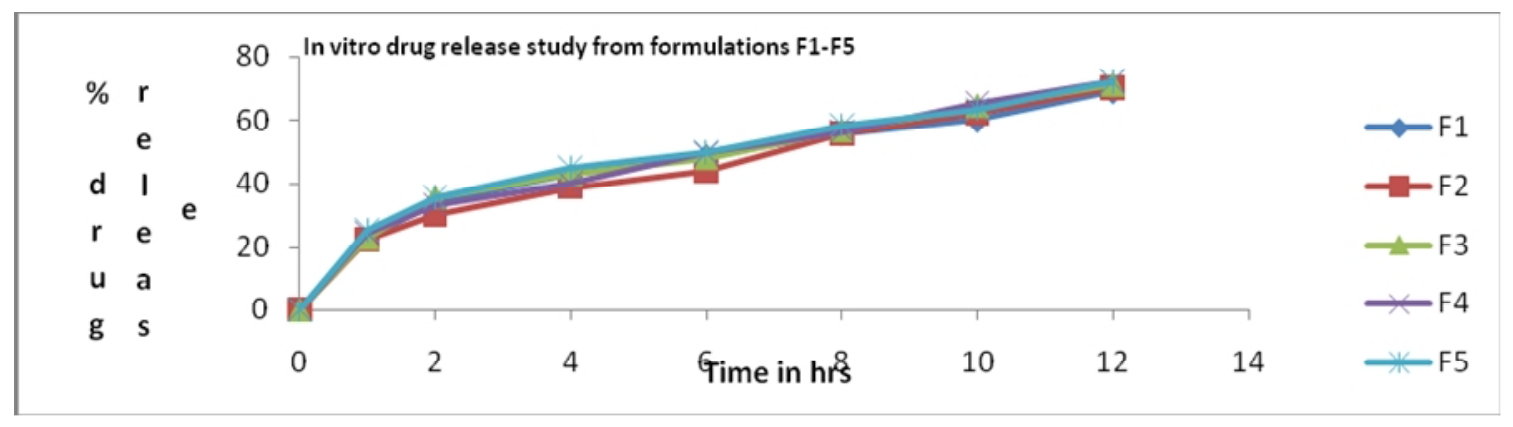

Figure 1

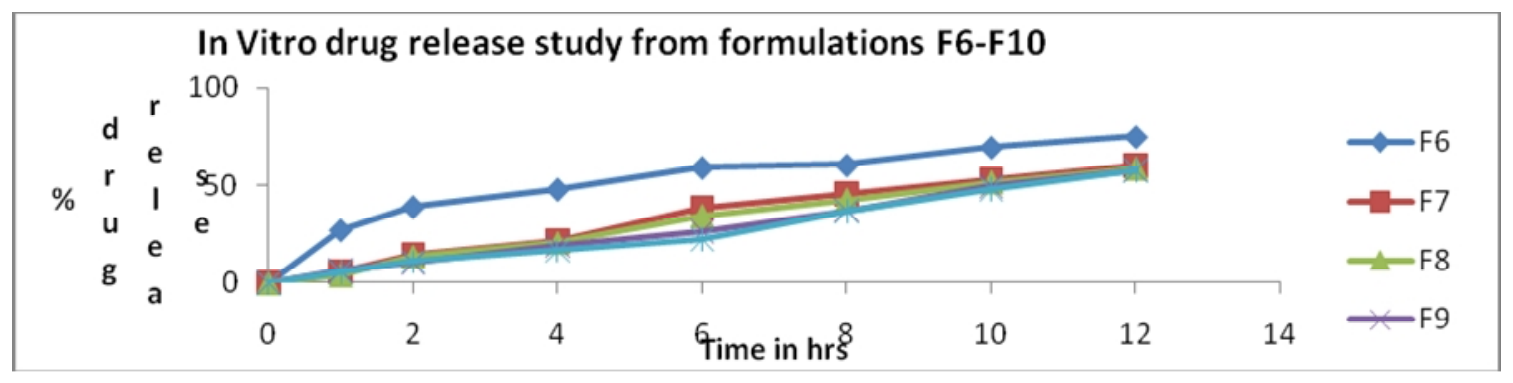

Figure 2

The results of angle of repose $(<25)$ indicated good flow properties of the entire granule blend. The compressibility index value were recorded, result in good excellent flow properties.

Formulated powder blends density; Porosity and hardness are often interrelated properties and are likely to influence compressibility, porosity, dissolution profile and properties of tablets made from it. The results of percentage porosity indicating that packaging of the granule blend may range from close to lose packaging and also confirming that particle are not of greatly different sizes. All the results indicate that the formulated granule blend possessing satisfactory flow properties and compressibility.

The tablets of different formulations (F1 to F10) were evaluated for various parameters they are thickness, diameter, hardness, friability, percentage weight variation percentage drug content and dissolution studies. All the formulations showed uniform thickness and diameter. In a weight variation test, the pharmacopeias limit for the percentage deviation for the tablets of more than $350 \mathrm{mg}$ is $\pm 5 \%$. The average percentage deviation of all tablet formulations was found to be within the above limit and hence all formulations passed the test for uniformity of weight as per official requirements. Drug content was found to be uniform among different batches of the tablets and the percentage of the drug content was more than $96 \%$. The hardness of all the formulation was between 4.0 to $5.5 \mathrm{~kg} / \mathrm{cm}^{2}$. The Percentage friability for all formulations was below $1 \%$ indicating that the friability is within the prescribed limits. The percentage drug release is found to be $75.02 \%$. All the tablet formulations showed pharmacotechnical properties and complied with specifications for weight variation, drug content, hardness and friability shown in the table $2 \& 3$.

\section{In vitro buoyancy studies}

The floating behavior studies it was found that as the concentration of effervescent mixture increase, the floating lag time, floating duration and matrix integrity decreased and vice versa were observed through results. A reverse trend was observed on increasing the polymer concentration. The Initial batch is prepared without sodium bicarbonate did not show any sign of floating. Therefore, sodium bicarbonate was used as a gas generating agent in order to float the tablet. The sodium bicarbonate induces $\mathrm{Co} 2$ generation in the presence of dissolution medium $(0.1 \mathrm{~N} \mathrm{HCl})$. The gas generated is trapped and protected within the gel formed by hydration of the polymer, thus decreasing the density of the tablet below $1 \mathrm{gm} / \mathrm{ml}$, and the tablet become buoyant. To study the effect of sodium bicarbonate concentration on floating lag tie batches F1 to F10 were selected. It was found that as the amount of sodium bicarbonate increases the floating lag time decreases. Thus sodium bicarbonate $30 \mathrm{mg}$ was essential to achieve optimum in vitro buoyancy (i.e floating lag time of 45 minutes and floating duration of $12 \mathrm{hrs}$ ). Further increase in the concentration of sodium carbonate does not show any significant effect of floating behavior. The increased amount of sodium bicarbonate caused a large amount of effervescence, which in turn resulted in pore formation, which led to rapid drug release. Thus $30 \mathrm{mg}$ concentration of sodium bicarbonate was kept constant for batches F5 to F10, which showed floating lag time between 4 and 5 minutes and remained floating for more than 12 hours. The relationship between the amounts of gas generating agents and floating lag time as well as the duration of floating are shown in table. It was observed that floating lag time for this system in the range of 4 to 15 min and flotation was achieved maximum at gas generating quantity of $30 \mathrm{mg}$ with in $4 \mathrm{~min}$. Therefore the formulation F5 to F10 was selected for In-vitro Dissolution study of floating matrix tablet. as shown in the table 4 .

\section{In vitro dissolution studies}

Depending on floating lag time and total floating time formulation F5 to F10were selected for in vitro dissolution study. In formulations F5 to F10 were showed $72.21 \%$, $75.02 \%, 59.85 \%, 58.52 \%, 58.07 \%$ and $57.96 \%$ at the end $12^{\text {th }}$ hour. Incorporation of higher amounts of cetyl alcohol in 
formulation F7, F8, F9 and F10 was found to be less drug release characteristics due to excess of cetyl alcohol in comparison F5 and F6. The drug released from the formulations diffusion coupled with erosion. Formulation F6 is selected as optimized formulation among all the formulations showing $75 \%$ sustained release at the end of 12 hours as shown in following figures $1 \& 2$.

\section{Compatibility studies of infra red}

IR spectra of drug diclofenac sodium and optimized formulation F5 (drug and Polymer mixture) were shown in tables and figures. The studies revealed that there was no significant interaction between drug and polymer.

\section{CONCLUSION}

The present study was to develop an floating system of diclofenac sodium with the use of wax materials, swell able polymer, release retardant and alkalizing agent which proved to be an ideal formulation, as it released the drug in a controlled manner for extended period of time by maintaining the buoyancy. The optimized formulation F5 reveals that in terms of floating lag time (5minurtes) and floating duration of 12 hours and drug release more than $75 \%$ at the end of 12 hrs. This results in developing, because a longer gastric residence time is an important condition for higher bioavailability of the drugs included in floating matrix dosage forms.

\section{REFERENCES}

1.Arora S, Ali J, Ahuja A, Khar RK, Baboota S."floating drug delivery systems: a review". Aaps pharmscitech. 2005; 06 (03): 372-388. http://dx.doi.org/10.1208/pt060347

2. Mavanshi AV and Gajjar SS. "Floating drug delivery systems to increase gastric retention of Drugs : A Review, Research J.Pharm and Tech., OctDec. 2008:347.

3. Patel Geeta M Patel Hitesh R, Patel Madhabhai " Floating Drug Delivery system: An Innovative approach to prolong gastric retention" www.pharmainfo.net, 2007; 5 (6).

4. Indian Pharmacopoeia, Government of India, Ministry of Health and Family welfare, The Indian Pharmacopoeia commission, Ghaziabad, 2007; II: $1020-1022$.

5. U.S. Pharmacopoeia NF 2004, Asian edition,United states Pharmacopeal Convention, INC., Rockville, 2004: 593-596.

6. Britsh Pharmacopoeia, 2003, 4 th edition, the stationary office, London, 2003; 1 : 605-606.

7. The Merck Index, fourteenth edition, Merc \& Co., Inc., USA, 2006:522.

8. Martindale, The Complete Drug Reference, $36^{\text {th }}$ edition, Pharmaceutical Press, London, 2009:44-47.

9. Satoskar R.S., Bhandrakar S.D., Ainapure S.S., Pharmacology and Pharmacotherapeutics, Eighteen edition, Popular Prakashan, Mumbai 2003: 169-170.

10. Remington: The Science and Practice of Pharmacy, Twentieth edition, Philadelphia,

2000; II: 1456

11. Kumar Ravi, Patil Swati, Patil B. M.,. Patil Sachin R, Mahesh S. Paschapur, "Design and In vitro Evaluation of Oral Floating Matrix Tablets of Aceclofenac" International Journal of Chem.Tech Research, Oct-Dec 2009; 1 (4):815-825.

Cite this article as:

Subramaniyan Chandra, S.Gopi, Biyogo ogetii alex, Omarbakri nasreidin elsayed, Oleti Chandra sai pavan. Design and in vitro evaluation studies of Diclofenac sodium floating matrix tablets by melt granulation technique. Int. Res. J. Pharm. 2013; 4(5):150-154 